\title{
Discrete-Time LQ Optimal Repetitive Control
}

\author{
Hakan Köroğlu Ömer Morgül \\ Bilkent University, Dept. Electrical \& Electronics Engineering, Bilkent, 06533, Ankara, Turkey. \\ Corresponding author : H. Köroğlu, Tel : (90-312) 290-2384, Fax : (90-312) 266-4307, E-mail : korogludee.bilkent.edu.tr
}

\begin{abstract}
LQ optimal repetitive control is developed in singleinput single-output discrete-time signal/system framework. For a given plant and a stabilizing controller, the LQ optimal repetitive control system can be obtained by the addition of a plug-in unit to the existing control system. The overall behaviour (stochastic behaviour, stability robustness etc.) of the new system can be improved by the appropriate choice/tuning of the design parameters.
\end{abstract}

Keywords : Control design, LQ control, Repetitive control.

\section{Introduction}

Various real-life systems are subject to periodic disturbances. Moreover in automation systems, repetitive tasks come into picture. These facts led to the evolution of a new area of study named repetitive control, in the beginning of $80 \mathrm{~s}$. The basic aim in repetitive control system design is the achievement of tracking/rejection goals in a periodic signal framework in which the period of the signals is known. For an extensive summary of results in this area of research, the reader is referred to the recent survey of (Hillerstrom and Walgama, 1996) and the references cited therein.

After the preceding continuous-time formal presentation of the repetitive system design by (Hara et al, 1988), the design is considered for discrete-time systems in (Tomizuka et al, 1989) and a direct solution is presented based on the zero phase error tracking approach of (Tomizuka, 1987). This is then modified in (Chew and Tomizuka, 1990) by considering the robustness and stochastic behaviour aspects. Though pointed out in (Hara et al, 1988) and (Chew and Tomizuka, 1990 ), the case of optimal design is not fully elaborated. In (Peery and Özbay, 1997), $\mathcal{H}_{\infty}$ optimal control is considered in repetitive control framework.

Due to the undesirable effects of high power control inputs in control systems, Linear Quadratic (LQ) optimal control approach is used to achieve imperfect tracking/rejection with desirably less control effort. In this work, we consider the LQ optimal design for the case in which the reference/disturbance signals are known to be periodic. In the next section, we summarize the discrete-time repetitive control structures of (Tomizuka et $a l, 1989)$ and (Chew and Tomizuka, 1990). In Section 3 , we develop a $L Q$ optimal repetitive controller and a plug-in type implementation of the proposed controller. We also discuss the appropriate choice of the design parameters for better transient performance, steady-state behaviour and stability robustness. Section 4 illustrates the effects of the design parameters with the help of several simulations. The paper ends with some concluding remarks.

\section{Discrete-Time Repetitive Control}

Throughout the paper, we consider the single-input single-output discrete-time systems in a linear timeinvariant (LTI) framework. We assume a plant with the input/output relation

$$
y(t)=P(z) u(t)+d(t),
$$

where $P$ is a causal rational transfer function, and $u, y$ and $d$ are the plant input, output and the disturbance signals respectively. With the one degree of freedom control system of Figure 1, the control input supplied to the plant is given by

$$
u(t)=C_{\mathrm{FB}}(z) e(t),
$$

where $C_{\mathrm{FB}}$ is the transfer function of the LTI feedback controller and $e$ is the tracking error defined as

$$
e(t)=r(t)-y(t),
$$

with $r$ being the reference signal to be tracked. With the sensitivity of the closed loop of Figure 1 defined as

$$
S_{\mathrm{CL}}(z)=\left[1+P(z) C_{\mathrm{FB}}(z)\right]^{-1},
$$

it is straightforward do derive that

$$
e(t)=S_{\mathrm{CL}}(z)(r(t)-d(t)) .
$$

A basic concern in control system design is to keep the tracking error small by the choice of an appropriate controller, which should necessarily keep the sensitivity function small. This choice can be simplified by 


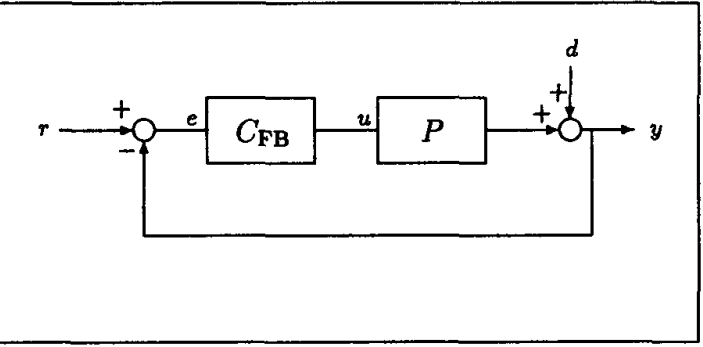

Figure 1: Unity feedback control system.

leaving aside the most general case and assuming special reference/disturbance signals. Repetitive control considers the case in which the signals are assumed to be periodic with a known period. The basic principle used to develop repetitive control schemes is the $I n$ ternal Model Principle, which is satisfied in this case by the inclusion of the delayed positive feedback structure in the feedback loop. The transfer function of this structure for a period of $n$ is given by

$$
C_{\mathrm{dprb}}(z)=\frac{1}{1-z^{-n}}
$$

Discrete-time periodic signals contain finitely many frequencies in the interval $[0,2 \pi]$ and for a signal of period $n$ they are given by

$$
\omega_{i}=\frac{2 \pi}{n} i ; \quad i=0,1, \ldots, n-1 .
$$

The values of $C_{\mathrm{dprb}}\left(\omega_{i}\right)$ are infinity which means that a closed loop including this transfer function will have perfect tracking/rejection performance for periodic reference/disturbance signals, provided that the closed loop is stable. Preserving stability is not trivial and hence the choice of the overall feedback controller is important. (Tomizuka et al, 1989) proposed their zero phase error tracking controller (ZPETC), as the accompanying part of the delayed positive feedback structure. ZPETC was developed in (Tomizuka, 1987) for feedforward tracking purposes. If the transfer function of a stable and causal plant is denoted as

$$
P(z)=\frac{N_{P}(z)}{D_{P}(z)},
$$

with $N_{P}$ and $D_{P}$ being coprime numerator/denominator polynomials, the ZPETC (that is also suited for use in repetitive controller structure) is given by

$$
C_{\mathrm{ZP}}(z)=\frac{D_{P}(z) N_{P}^{-}\left(z^{-1}\right)}{\left\|N_{P}^{-}\right\|_{\infty}^{2} N_{P}^{+}(z)}
$$

Here the superscripts + and - are used to denote the stable (i.e having zeros inside the unit circle) and unstable (i.e having zeros on or outside the unit circle) parts of the polynomials respectively (i.e. $N_{P}(z)=$ $\left.N_{P}^{+}(z) N_{P}^{-}(z)\right) .\|H\|_{\infty}$ is used to denote the well-known $\mathcal{H}_{\infty}$ norm of $H$ which is defined in our framework as

$$
\|H\|_{\infty}=\sup _{\omega \in[0,2 \pi)}|H(\omega)| .
$$

During feedforward application, the reference to be tracked should be known in advance due to the noncausality of $C_{\mathrm{ZP}}$. In (Tomizuka et al, 1989), a prototype discrete-time repetitive controller is formed as the multiple of $C_{\mathrm{dpfb}}$ and $C_{\mathrm{ZP}}$ with a scalar and $n$ step delay, to satisfy perfect tracking/rejection for periodic reference/disturbance signals respectively. Due to stability robustness and stochastic behaviour considerations, this structure is modified in (Chew and Tomizuka, 1990), by modifying the delayed positive feedback structure as

$$
C_{\mathrm{dpfb}}^{\mathrm{mod}}(z)=\frac{F_{\mathrm{dpfb}}(z)}{1-F_{\mathrm{dpfb}}(z) z^{-n}}
$$

where $F_{\mathrm{dpfb}}$ is a filter (preferably of low-pass nature due to robustness considerations) which satisfies $\left|F_{\mathrm{dpfb}}(\omega)\right| \leq 1$. The modified repetitive controller is then formed as

$$
C_{\mathrm{ZP}}^{\mathrm{rep}}(z)=k_{\mathrm{rep}} z^{-n} C_{\mathrm{dprb}}^{\mathrm{mod}}(z) C_{\mathrm{ZP}}(z) .
$$

The control system of Figure 1 is stable with $C_{\mathrm{FB}}=$ $C_{\mathrm{ZP}}^{\text {rep }}$ if $k_{\text {rep }} \in(0,2)$ and the plant is stable. The unmodified structure (which supplies perfect tracking/rejection) can be obtained with $F_{\mathrm{dprb}}(z)=1$.

\section{LQ Optimal Repetitive Control}

Perfect satisfaction of tracking/rejection goals in control systems might not be possible or desirable due to the need for high power control inputs. This is because high power control inputs might cause actuator saturation. Also it is desirable to keep the cost low, which typically necessitates low power control inputs. These design considerations are included in the well-known area of Linear Quadratic (LQ) control. The infinite horizon (or steady-state) frequency weighted LQ cost is defined in SISO discrete-time signal/system framework as (see (De Bruyne et al, 1995))

$$
J_{\mathrm{LQ}}=\lim _{\tau \rightarrow \infty} \frac{1}{\tau} \sum_{t=0}^{\tau-1}[e(t)]^{2}+[F(z) u(t)]^{2},
$$

where $F(z)$ is a stable LTI filter. By minimizing the LQ cost, the plant output is forced to follow the reference command in a quadratically optimal sense while keeping the power of the filtered control input at a desired level. The level of penalization on the power of the control input is determined by the frequency response of $F$ and the case of $F=0$ corresponds to the quadratically optimal perfect tracking/rejection. 
If the control system of Figure 1 is assumed to be internally stable and if, moreover, $r-d$ is a quasi-stationary signal with power spectrum $\Phi(\omega)$, the LQ cost defined by (12) can equivalently be evaluated in the frequency domain according to

$$
J_{\mathrm{LQ}}=\frac{1}{2 \pi} \int_{0}^{2 \pi} j(\omega) \Phi(\omega) d \omega,
$$

with

$j(\omega)=\left.\left.\left[1-|G(\omega)|^{2}\right]^{-1}\left|S_{C L}(\omega)-\right| G(\omega)\right|^{2}\right|^{2}+|G(\omega)|^{2}$,

where $G$ is the solution of the spectral factorization equation

$$
G(z) G\left(z^{-1}\right)=\frac{F(z) F\left(z^{-1}\right)}{F(z) F\left(z^{-1}\right)+P(z) P\left(z^{-1}\right)} .
$$

As easily noted, $j(\omega) \Phi(\omega)$ here serves as the cost contribution corresponding to frequency $\omega$. This means that the frequency domain formula can be useful for the case of a spectrum consisting of impulses at finitely many frequencies. Periodic signals constitute such a family for which the following optimality condition can be derived.

Theorem 1 If $r-d$ is a periodic signal (which will be the case if $r$ and $d$ are both periodic) with period $n$, then the control system of Figure 1 is LQ optimal if and only if it is stable and the feedback controller satisfies

$$
C_{\mathrm{FB}}\left(\omega_{i}\right)=\frac{P^{*}\left(\omega_{i}\right)}{\left|F\left(\omega_{i}\right)\right|^{2}},
$$

where $\omega_{i}$ are the harmonics given by (7). (If $P\left(\omega_{i}\right)=$ $\infty$ or 0 then that $i$ should be removed.)

The basic motivation in the introduction of the modified delayed positive feedback structure of $(10)$ in place of (6) was the improvement of the control system robustness (see (Tsao and Tomizuka, 1988)). The effect of this modification on the control input will be dependent on the choice of the filter $F_{\mathrm{dpfb}}$. Though the choice of $F_{\text {dpfb }}$ is formalized in (Tomizuka, 1993) (a low-pass filter of zero-phase nature is proposed), LQ optimality issue is not considered. It can be shown that the modified repetitive controller of (11) satisfies an LQ optimality criterion for stable plants with an appropriate choice of $F_{\mathrm{dpfb}}$. For the case of unstable plants, the plant should first be stabilized and the repetitive controller should be designed using the overall transfer function of the stabilized loop. In this case, LQ optimality condition will, most probably, be violated.

In this section, we propose a controller which is LQ optimal for both stable and unstable plants with periodic signals under consideration, and analyze its overall performance. The leading idea is to use the delayed positive feedback structure of (6) to satisfy the LQ optimality condition of (16) at the harmonics $\omega_{i}$. We first assume a causal and stabilizing controller $(C)$ of the form

$$
C(z)=\frac{N_{C}(z)}{D_{C}(z)}
$$

where $N_{C}$ and $D_{C}$ are coprime polynomials. The characteristic polynomial of the closed loop formed by $P$ and $C_{\mathrm{FB}}=C$ (see Figure 1 ) is then given by $Q$, where

$$
Q(z)=N_{P}(z) N_{C}(z)+D_{P}(z) D_{C}(z) .
$$

The fact that $C$ is stabilizing is equivalent to stating that $Q$ is stable. In order to determine a stabilizing controller for a given plant, equation (18), which is known as the Diophantine equation, is to be solved for some stable $Q$. Similarly, we assume that the transfer function of the frequency shaping filter $F$ is given by

$$
F(z)=\frac{N_{F}(z)}{D_{F}(z)},
$$

where $N_{F}$ and $D_{F}$ are polynomials which are both stable and coprime. We, moreover, assume that $F$ is causal. Using the notation

$$
H^{*}(z)=H\left(z^{-1}\right),
$$

and dropping the $z$ dependency of the polynomials, we can represent a controller satisfying the LQ optimality condition given by (16), as

$C_{\mathrm{LQ}}^{\mathrm{rep}}(z)=\frac{k_{\mathrm{rep}} D_{F} D_{F}^{*} N_{P}^{*} M M^{*} Q+N_{C}\left(z^{n}-1\right)}{k_{\mathrm{rep}} N_{F} N_{F}^{*} D_{P}^{*} M M^{*} Q+D_{C}\left(z^{n}-1\right)}$.

Here $M$ is a stable polynomial which is not identically equal to zero, and $k_{\text {rep }}$ is a constant which we will call as the repetitive control gain, in accordance with the other works (see (Tomizuka et al, 1989) and (Chew and Tomizuka, 1990)).

In accordance with (15), we define the polynomials $N_{G}$ and $D_{G}$ as the stable solutions of the spectral factorization equations

$$
\begin{aligned}
& N_{G} N_{G}^{*}=N_{F} N_{F}^{*} D_{P} D_{P}^{*} \\
& D_{G} D_{G}^{*}=N_{F} N_{F}^{*} D_{P} D_{P}^{*}+D_{F} D_{F}^{*} N_{P} N_{P}^{*}
\end{aligned}
$$

The characteristic polynomial of the feedback system formed by $P$ and $C_{\mathrm{FB}}=C_{\mathrm{LQ}}^{\text {rep }}$ is given by

$$
Q_{\mathrm{LQ}}^{\mathrm{rep}}=z^{n} Q(z) Q_{\mathrm{rep}}(z) \text {, }
$$

where

$$
Q_{\mathrm{rep}}(z)=1+z^{-n}\left[k_{\mathrm{rep}} M M^{*} D_{G} D_{G}^{*}-1\right] .
$$

The following theorem gives a similar stability condition to that of zero phase error controller of (11). 
Theorem 2 Consider the unity feedback control system of Figure 1, with the transfer function of the plant given by (8). Let the closed loop with $C_{\mathrm{FB}}=C$, where $C$ is given by (17), be internally stable. The feedback system formed by $P$ and $C_{\mathrm{LO}}^{\text {rep }}$ is internally stable for $0<k_{\text {rep }}<2$ and is $L Q$ optimal for periodic reference/disturbance signals of period $n$, if $M$ is chosen to satisfy

$$
\left\|M D_{G}\right\|_{\infty}=1 \text {. }
$$

In order to design an LQ optimal controller for a given plant, the Diophantine equation of (18) is to be solved for some arbitrary stable polynomial $Q$. An LQ optimal controller can also be obtained by the polynomial approach to the LQ problem (see ( $\AA$ ström and Wittenmark, 1997)), however this approach necessitates the solution of a spectral factorization problem and a more complex Diophantine equation. Also the knowledge of the spectrum of the signal is necessary. The above approach, on the other hand, has the flexibility of choosing the stable polynomial $Q$. If a controller is known to be stabilizing, $Q$ can directly be found with the help of (18). The case of a stable plant thus possesses a direct solution which can be obtained with $N_{C}=0, D_{C}=1$ and $Q=D_{P}$.

There are infinitely many possible choices for $M$ which can satisfy the stability condition given by (25), and this is helpful in the sense that the overall performance (convergence, stochastic behaviour and robustness) of the LQ optimal repetitive controller can be improved by appropriate choices. A trivial choice for $M$ can be seen to be $M=\left\|D_{G}\right\|_{\infty}^{-1}$. A more preferrable one is a polynomial approximating $D_{G}^{-1}$. This choice prevents an arbitrary variation for $Q_{\text {rep }}(\omega)$ which is important for robust stability.

\subsection{Implementation}

With $n_{P}=\operatorname{deg} D_{P}, n_{C}=\operatorname{deg} D_{C}, n_{F}=\operatorname{deg} D_{F}$, $n_{M}=\operatorname{deg} M$ and $n_{Q}=n_{P}+n_{C}$, we can rewrite the controller of (20) as

$$
C_{\mathrm{LQ}}^{\mathrm{rep}}(z)=\frac{z^{n_{C}} D_{C}^{-1}\left[z^{-n_{C}} N_{C}+k_{\mathrm{rep}} z^{-\delta} C_{\mathrm{dpfb}} F_{\mathrm{rl}} F_{\mathrm{ff}}\right]}{1+z^{n c} D_{C}^{-1} k_{\mathrm{rep}} z^{-\delta} C_{\mathrm{dpfb}} F_{\mathrm{rl}} F_{\mathrm{fb}}},
$$

where

$$
\delta=n-n_{P}-n_{F}-n_{M},
$$

and

$$
\begin{aligned}
& F_{\mathrm{rl}}(z)=z^{-n_{M}-n_{Q}} Q M M^{*} \\
& F_{\mathrm{ff}}(z)=z^{-n_{F}} D_{F} D_{F}^{*} N_{P}^{*}, \\
& F_{\mathrm{fb}}(z)=z^{-n_{F}} N_{F} N_{F}^{*} D_{P}^{*} .
\end{aligned}
$$

If $C$ is causal (i.e $\operatorname{deg} N_{C} \leq \operatorname{deg} D_{C}$ ) and $\delta \geq 0$ (This condition does not pose any restriction as in the discrete-time signal/system framework it can be satisfied by viewing the period as $2 n, 3 n$ or as whatever

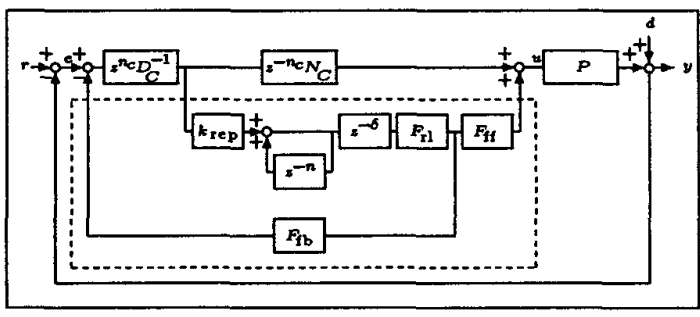

Figure 2: LQ optimal repetitive control system.

appropriate. If the design considers a sampled-data system, then the sampling can be done accordingly.), this controller can be realized by the addition of a plugin unit to the stable closed loop formed by $P$ and $C$ as in Figure 2.

\subsection{Steady-State and Stochastic Behaviour}

With the sensitivity defined as in (4), we can obtain $S_{\mathrm{LQ}}^{\text {rep }}$ as

$$
S_{\mathrm{LQ}}^{\mathrm{rep}}(z)=\frac{\left(1-z^{-n}\right) S+k_{\mathrm{rep}} z^{-n} M M^{*} N_{G} N_{G}^{*}}{Q_{\mathrm{rep}}}
$$

where $S$ denotes the sensitivity of the stabilized loop (i.e. $S=[1+P C]^{-1}$ ). Obviously, the sensitivity at the harmonics $\omega_{i}$ are given by $\left|G\left(\omega_{i}\right)\right|^{2}$. As the systems under consideration will have unavoidable stochastic disturbances in addition to repetitive disturbances, large magnitude is not desirable for the sensitivity function. If $M$ is chosen to satisfy $M \approx D_{G}^{-1}, S_{\mathrm{LQ}}^{\text {rep }}$ can be kept close to $S$ with a small (i.e. close to zero) choice for $k_{\text {rep. }}$. This means that the overall sensitivity variation in the repetitive system can be forced to resemble the overall sensitivity variation in the stabilized loop, the design of which is at our disposal.

\subsection{Stability Robustness and Transient Be- haviour}

With the design polynomial $M$ chosen to approximate $D_{G}^{-1}$, all zeros of $z^{n} Q_{\text {rep }}$ will approximately be zero with $k_{\text {rep }}=1$. If the zeros of $Q$ are far away from the unit circle, convergence to steady-state will be fast. Moreover the system will be robust due to the prevention of arbitrary frequency variation for $Q_{\text {rep }}$. For multiplicative plant perturbations, the complementary sensitivity function (defined as $T_{\mathrm{CL}}=1-S_{\mathrm{CL}}$ ) will indicate the degree of robustness. As described above LQ optimal system complementary sensitivity $\left(T_{\mathrm{LQ}}^{\text {rep }}\right)$ can be kept close to stabilized sytem complementary sensitivity $(S)$ with a small $k_{\text {rep. }}$ Yet an unstable pole/zero cancellation is approached as $k_{\text {rep }}$ gets closer to zero. Thus it might be preferrable to tune $k_{\text {rep }}$ to unity and increase the stability robustness by appropriate choice of $F$ in the LQ cost. 


\section{Example Simulations}

In this section we present the results of several simulations realized with the stable nonminimum phase plant described by

$$
P(z)=\frac{z-2.5}{z^{2}-1.4 z+0.45} .
$$

The reference $(r)$ is set to zero and the disturbance is generated as the superposition of a periodic signal and stochastic signal (i.e. $d(t)=d_{\mathrm{p}}(t)+d_{\mathrm{s}}(t)$ ). Periodic disturbance is arbitrarily generated and has a period of 20 and variance of unity, wheras the stochastic disturbance is generated from a white noise process $(w)$ of variance 0.05 as

$$
d_{\mathrm{s}}(t)=\frac{z^{2}-0.2 z+0.2}{z^{2}-1.4 z+0.45} w(t) .
$$

LQ optimal repetitive controller design is considered for

$$
F=2-z^{-1},
$$

and $M$ is chosen (to approximate $D_{G}^{-1}$ ) as

$M=0.2444 z^{4}+0.2228 z^{3}+0.1192 z^{2}+0.0458 z+0.0134$.

In Figure 3, we give the simulation results for several different choices of $k_{\text {rep }}$ and $C . C_{\mathrm{MV}}$ is the minimum variance controller for $d(t)=d_{s}(t)$, which can be found to be (see (Åstrom and Wittenmark, 1997))

$$
C_{\mathrm{MV}}(z)=\frac{-0.6047 z+0.2848}{z+1.4047} \text {. }
$$

Simulation results indicate that the choice of the design parameters can significantly improve the quality of the overall design. Best response to stochastic disturbance is obtained with $k_{\text {rep }}=0.1$ and $C=C_{\mathrm{MV}}$. This is because the closed loop behaviour for this case resembles the closed loop behaviour with the minimum variance controller. Yet the convergence to steady-state is slow as $k_{\text {rep }}$ is not close to unity.

\section{Conclusions}

We considered the LQ optimal design of repetitive controllers for discrete-time systems. Given an arbitrary stabilizing controller, the $\mathrm{LQ}$ optimal repetitive controller can directly be obtained. For stable plants, knowledge of the plant is enough for the design. With a sufficiently large period, the repetitive system can be obtained by the addition of a plug-in unit to the stabilized system. The proposed controller structure can be used to design repetitive control systems with improved robustness and stochastic behaviour by appropriate choice/tuning of the design parameters. Adaptive and optimal tuning of the design parameters is supposingly a promising direction for further research. Extending the development to include continous-time systems is a standard but nontrivial research problem.

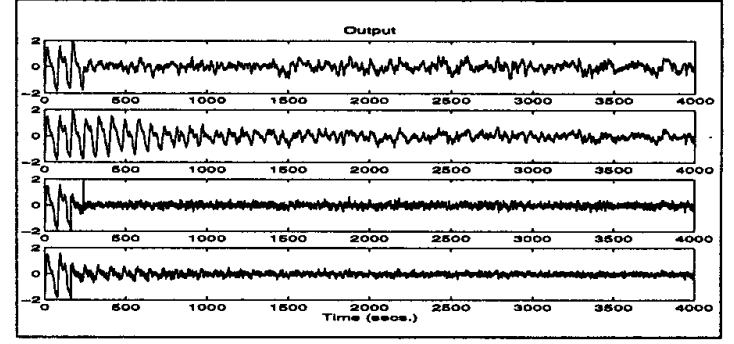

Figure 3: Stochastic behaviour (top: $C=0, k_{\text {rep }}=1.0$; middle-1: $C=0, k_{\text {rep }}=0.1$; middle-2: $C=C_{\mathrm{MV}}$, $k_{\text {rep }}=1.0$; bottom: $C=C_{\mathrm{MV}}, k_{\text {rep }}=0.1$ ).

\section{References}

[1] K. J. Åström and B. Wittenmark. Computer Controlled Systems : Theory and Design. Prentice Hall, Upper Saddle River, NJ, 1997.

[2] F. De Bruyne, B. D. O Anderson, M. Gevers and J. Leblond. LQG Control With Pole Constraints Using the Youla Parametrization. Proc. of 3rd European Control Conf., pp.2494-2499, Rome, Italy, Sept. 1995.

[3] K. K. Chew and M. Tomizuka. Steady-State and Stochastic Performance of a Modified Discrete-Time Prototype Repetitive Controller. ASME Journ. of Dynamic Systems, Measurement and Control, 112(1):3541,1990 .

[4] S. Hara, Y. Yamamoto, T. Omata, and M. Nakano. Repetitive Control System : A New Type Servo System for Periodic Exogenous Signals. IEEE Trans. on Automatic Control, 33(7):659-668, 1988.

[5] G. Hillerström and K. Walgama. Repetitive Control Theory and Applications - A Survey. Proc. of 13th IFAC World Congr., Vol.D, pp.1-6, San Francisco, USA, June 1996.

[6] T. E. Peery and H. Özbay. $\mathcal{H}_{\infty}$ Optimal Repetitive Controller Design for Stable Plants. ASME Journ. of Dynamic Systems, Measurement and Control, 119(3):541-547, 1997.

[7] M. Tomizuka. Zero Phase Error Tracking Algorithm for Digital Control. ASME Journ. of Dynamic Systems, Measurement and Control, 109(1):65$68,1987$.

[8] M. Tomizuka. On the Design of Digital Tracking Controllers. ASME Journ. of Dynamic Systems, Measurement and Control, 115(2B):412-418, 1993.

[9] M. Tomizuka, T. C. Tsao, and K. K. Chew. Analysis and Synthesis of Discrete-Time Repetitive Controllers. ASME Journ. of Dynamic Systems, Measurement and Control, 111(3):353-358, 1989.

[10] T. C. Tsao, and M. Tomizuka. Adaptive and Repetitive Digital Control Algorithms for Noncircular Machining. Proc. American Control Conf., pp. 115-120, Atlanta, GA, June 1988. 